\title{
Instrumentally based conditioned avoidance response acquisition in goldfish in a simultaneous presentation task
}

\author{
D. J. ZERBOLIO, JR., and L. L. WICKSTRA \\ University of Missouri, St. Louis, Missouri 63121
}

\begin{abstract}
Goldfish, run in a Y-maze, with the simultaneous presentation of a cue associated with shock and a second cue associated with safety (no US), acquired a decided preference for the safe cue. These data are interpreted as representing a clear demonstration of stimulusspecific conditioned avoidance. Because earlier research has been challenged by unusual control performances, this instrumental learning conclusion is hesitantly drawn.
\end{abstract}

Steiner (1971) suggested that learning conclusions based on increases in shuttle rate in goldfish might be meaningless unless operant shuttle rate increases, which do not adapt out in a few sessions, are taken into account. Exactly this problem has plagued learning interpretations in single-stimulus designs requiring active responses, since comparable performance increases are shown by goldfish tested with instrumental or classical US presentation procedures, and by CS-only and timelapse controls as well (Scobie \& Fallon, 1974; Woodard \& Bitterman, 1973; Zerbolio \& Wickstra, $1978 \mathrm{a}, 1978 \mathrm{~b})$. But the problem of unusual activity in controls is not limited to active response designs in goldfish. In single-stimulus passive avoidance designs, although goldfish show a decrease in response compared to yoked controls during the "passive" CS (Woodard \& Bitterman, 1973), comparable response inhibition occurs without a CS as well (Zerbolio \& Wickstra, 1978c), making a stimulus-specific interpretation dubious at best. Another unexpected control performance turns up in the successive-presentation discrimination procedure. Woodard and Bitterman (1971) showed that goldfish would respond in increasingly more US paired time frames than unpaired time frames when the US paired time frame was indicated by one cue and the unpaired time frame indicated by a second cue. Zerbolio and Wickstra (1979) found comparable results but also found the same discriminated behavior pattern (i.e., goldfish responding in increasingly more US paired time frames than unpaired time frames) when the cue indicating the US paired time frame was omitted, making the procedure an explicitly unpaired pseudoconditioning design. Thus, the same discriminated pattern of response occurs either with or without a cue indicating the US paired time frame, making interpretations about responses associated with the US paired cue very difficult to draw. In each case, the interpretation problems stem from the unexpected activity shown by some control group.

Another way to handle the unusual activity problem may be to simply assume at the start that goldfish will be active and that the activity will increase over sessions, independent of what is done to them except, of course, for the passive procedure per se. The problem could be answered by a procedure that allows an assessment of learning independent of activity rate changes. The simultaneous-presentation procedure may offer such an alternative. With two cues and their associated consequences presented simultaneously on each trial, it is a simple matter to determine whether or not the goldfish acquires a preference between them. If the procedure produces a simple increase in activity independent of the cues and/or their associated consequences, there should be little difference in the frequency of initial cue selection. If, however, one cue is preferred over another, for whatever reason, a disproportionate choice pattern favoring one cue should develop. If the animal is active enough to swim to all cues on all trials, the initial choice data provide information about preference. If the animal does not respond at all, that is easily measured too. The present study represents an initial investigation to determine whether or not goldfish show a preferential response with the simultaneouspresentation procedure. For that purpose, a Y-maze, incorporating the essential features of the shuttlebox (Zerbolio \& Wickstra, 1978a, 1978b, 1978c) was constructed.

\section{METHOD}

\section{Subjects}

Ten 5- to 6-cm goldfish, obtained from Ozark Fisheries and housed in individual $7.5 \times 11.5 \times 12.5 \mathrm{~cm}$ deep aquaria, served as subjects. Housing was well aeriated and filtered, with both temperature $\left(21.1^{\circ} \mathrm{C}\right)$ and ph $(7 \pm .1)$ held constant. Animals were fed daily.

\section{Apparatus}

Two identical Y-mazes, constructed of $.635-\mathrm{cm}$ clear plastic, with three alleys $120 \mathrm{deg}$ apart, $8.7 \mathrm{~cm}$ wide, and $10 \mathrm{~cm}$ deep, and from the exact center $20 \mathrm{~cm}$ long, were constructed (all inside dimensions, see Figure 1). 


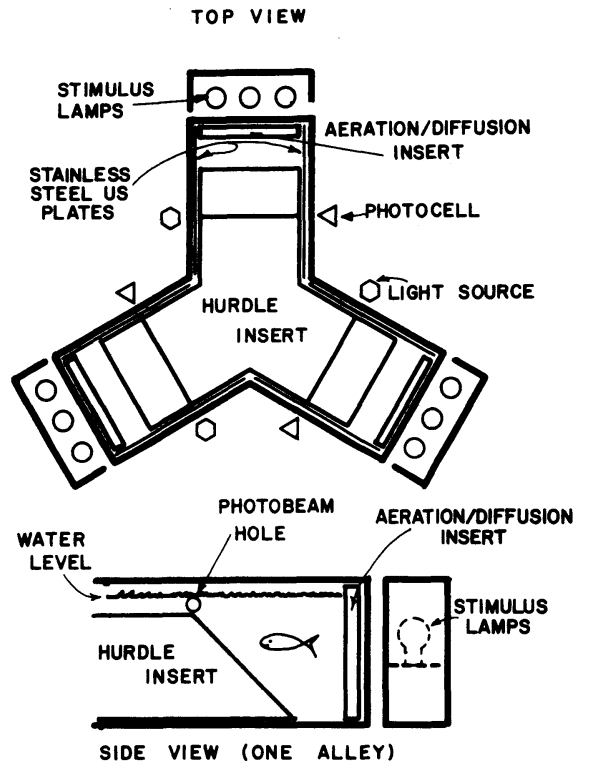

Figure 1. A stylized picture of a Y-maze for goldfish showing the relative placement of the essential elements. The dimensional details may be found under "Apparatus."

Each maze contained a three-projection 5-cm-high hurdle that extended $6.5 \mathrm{~cm}$ horizontally into each alley; from there the hurdle sloped at a 45-deg angle to the alley floor. The internal sides of each alley were lined with two $9 \times 16.5 \mathrm{~cm}$ 22-g stainless steel plates ( the photobeam and individual wires to provide US stimulation. Three photocell detectors, focused across the hurdle at the beginning of the 45-deg slope $(6.5 \mathrm{~cm}$ deep into each alley) served to monitor the subjects activity. The photocell light sources were G.E. 222 prefocused penlight bulbs run at approximately $1 \mathrm{~V} \mathrm{dc}$ to extend bulb life. Alley location of the subject was indicated by the last photobeam broken. At the inside end of each alley, a device to constantly aerate the water and to evenly diffuse end wall illumination was inserted. End wall illumination was provided by three $7-\mathrm{W} 110-\mathrm{V}$ ac Christmas tree bulbs located at the end of each alley (nine bulbs in all); each bulb was individually controlled and mounted and baffled so that light did not project on the other alleys. A clear plastic top on the maze prevented animals from jumping out. Water level was maintained at $7 \mathrm{~cm}(2 \mathrm{~cm}$ over the hurdle top $)$.

\section{Procedure}

All animals were run for 60 trials a day with a variableinterval (VI) 60-sec intertrial interval (ITI) for 10 consecutive days. During the ITI, all three alleys were illuminated with white $7-\mathrm{W} 110-\mathrm{V}$ ac light. At trial onset, the CS alley was the one occupied by the fish and the remaining two unoccupied alleys were the $S^{d}$ and $S^{\Delta}$ alleys. At trial onset, the white light was terminated in all alleys. Simultaneously, the $S^{d}$ stimulus (red for half the subjects, green for the remainder) occurred in one of the unoccupied alleys and the $S^{\Delta}$ stimulus (green for half and red for the remainder) occurred in the other unoccupied alley. Position (left or right relative to the subject in the occupied starting alley) of the $S^{d}$ occurred on half the trials to the subject's left and on the remainder to the subject's right in Gellerman series. The CS alley remained dark during the trial. If the subject did not swim to the Sd alley within the initial 10 -sec period of the trial, two $200-\mathrm{msec} 6-\mathrm{V}$ ac $(.695 \mathrm{~V} / \mathrm{cm})$ shock pulses, $.8 \mathrm{sec}$ apart, were administered. The US was delivered if the subject either stayed in the start alley or swam to the $S^{\Delta}$ alley. If, fol- lowing failure to arrive in the $S d$ alley in $10 \mathrm{sec}$, the subject received the first US pulse, it could escape the second US pulse by swimming to the Sd alley in the intervening .8 -sec period. If the subject swam to the $S^{d}$ alley or swam to the $S^{\Delta}$ alley and corrected its mistake by leaving and entering the $S d$ alley within $10 \mathrm{sec}$, the US was omitted. In the correction case, however, the initial response (to the $S^{\Delta}$ ) was considered an error. The CS, $S d$, and $S^{\Delta}$ stimulus conditions remained as set at the trial onset for the entire trial $(11.2 \mathrm{sec})$ and then were terminated simultaneously with the reonset of the white ITI illumination in all three alleys. Obviously, subjects' alley selection during the trial had only one behavioral consequence: whether or not the US was omitted. Under these conditions, the subject could choose not to respond, swim to the $S^{d}$, swim to the $S^{\Delta}$, or swim to both stimuli. In the latter class of responses, the animal would be avoiding all the time, but the responses would differ in that one class would be an initial response to $S^{\Delta}$ followed by an $S^{d}$ approach (which is a corrected response), whereas the second would be an $S^{d}$ response followed by an $S^{\Delta}$ response. Since the correction possibility existed, incidence of both of these response classes ( $S^{d}$ followed by $S^{\Delta}$ and $S^{\Delta}$ followed by $S^{d}$ ) were recorded as well as the incidence of $S^{d}$-only and $S^{\Delta}$-only responses. In addition, ITI shuttle responses (the number of times a subject left one alley and moved to another) were recorded. When the US was administered, it was delivered across the stainless steel plates lining only the starting and $S^{\Delta}$ alleys. This created a decreasing gradient of US intensity at the mouth of the $S^{d}$ alley. By using this US administration procedure, the subject was not as severely punished for a tardy approach to the Sd alley (at the time of US administration) as it was for other responses. All events and responses were programmed and recorded with automated equipment incorporating appropriate circuitry.

\section{RESULTS AND DISCUSSION}

Color of the stimuli (red or green) for $\mathrm{S}^{\mathrm{d}}$ and $\mathrm{S}^{\Delta}$ produced no reliable differences nor interacted with any other factor in the analyses. The data are presented in Figure 2.

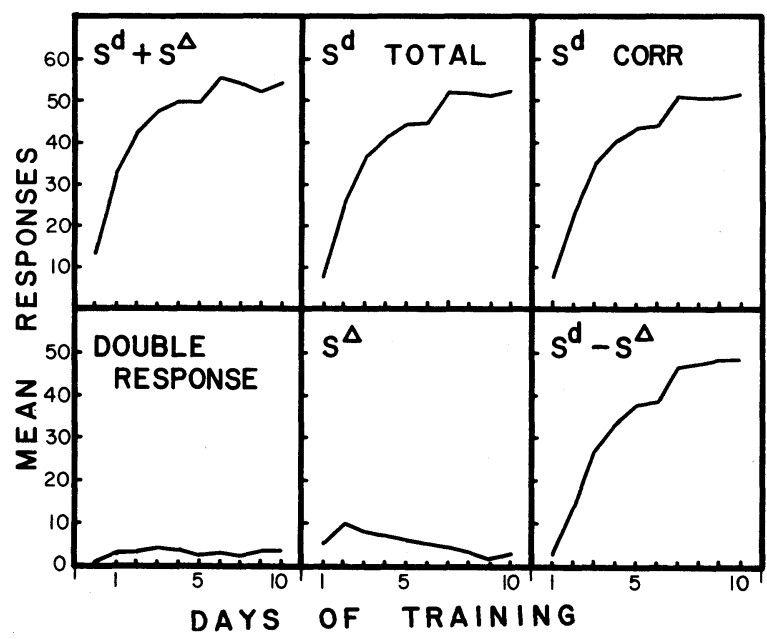

Figure 2. Goldfish performance in the $Y$-maze is shown, starting from the top left panel for the number of trials on which a response occurs $\left(S^{d}+S^{\Delta}\right)$, total avoidances ( $S^{d}$ total), initial $S^{d}$ choices only ( $S^{d}$ corr), number of trials where the subject visits both $S^{d}$ and $S^{\Delta}$ (double response), number of trials where the subject visits the $S^{\Delta}$ alley $\left(S^{\Delta}\right)$, and preponderance of $S^{d}$ over $S^{\Delta}$ in initial choice $\left(S^{d}-S^{\Delta}\right)$. 
The top left panel of Figure 2 shows that goldfish very quickly learned to respond to either the $S^{d}$ or $S^{\Delta}$. The first analysis considered the number of trials in which the goldfish arrived in the $S^{d}$ alley before the 10 -sec CS-US interval period and considered all $\mathrm{S}^{\mathrm{d}}$ responses, both initial and correction responses. As can be seen from the top middle panel of Figure 2, goldfish showed a substantial increase in the number of trials on which they swam to the $S^{d}$ over days of training $\left[F(9,72)=76.033, p<.01, \omega^{2}=.722\right]$. However, since an animal responding in an indiscriminate fashion could show the same performance level it if responded to both the $S^{d}$ and $S^{\Delta}$ stimuli in the 10-sec trial period before US onset, a second analysis was conducted on the number of trials where the animals initial response was to the $S^{d}$. Goldfish showed substantial increases in initial response rate to the $S^{d}$ with training $\left[F(9,72)=67.901, p<.01, \omega^{2}=.715\right]$. The initial $S^{d}$ responses are shown in the top right panel of Figure 2 as $S^{d}$ corr ( $S^{d}$ corrected).

As is already obvious, there is very little difference between the $S d$ total and $S^{d}$ corrected performance rates. But, since a very active animal could swim into both $S^{d}$ and $S^{\Delta}$ alleys on each trial without showing a preference, an analysis was done of the number of trials on which a double response occurred (either $S^{d}$ first, then $S^{\Delta}$ or $S^{\Delta}$ first, then $S^{d}$ ). Neither class of double response nor their total showed a change over days. The total double class incidence over days is shown in the lower left panel of Figure 2.

The rate of initial responses to the $\mathbf{S}^{\mathbf{\Delta}}$, which also includes the $S^{\boldsymbol{A}}$ then $S^{d}$ corrected responses, did show a change over days of training $[F(9,72)=3.481, p<.01$, $\left.\omega^{2}=.144\right]$, but, as is seen in the middle lower panel of Figure 2, this represents a decrease in $S^{\Delta}$ response of any kind after the second day of training.

Another analysis showing the increased preference of initial $S^{d}$ choice over $S^{\boldsymbol{A}}$ choices was calculated by taking $S^{d}-S^{\mathbf{A}}$ measures for each day for each animal. If goldfish respond indiscriminately, this analysis should represent no change with training, although the animals activity rate and response rate to both stimuli might increase. These data show a large increase in $S^{d}-S^{A}$ responses over days of training $\left[\mathrm{F}(9,72)=40.183, \mathrm{p}<.01, \omega^{2}=\right.$ .639], shown in the lower right panel of Figure 2 .

One further analysis on the mean ITI shuttle or ITI alley change rate found no change over days of training $[F(9,72)=1.512]$. The mean number of alley changes per day during the ITI was 45.9 .

From these data, it seems obvious that goldfish do not choose to remain in the start alley, but very rapidly learn to respond. Further, given that the choices available represent differential US consequences, goldfish show a decided preference for swimming to the Y-maze arm containing the cue that is associated with no-US presentation. And lastly, once having responded to the cue paired with no US, they do not seem predisposed to leave it, since the number of double responses seen is very low. Essentially, then, goldfish seem quite capable of actively selecting the safe (no-US) cue alternative when given the choice between a cue associated with US presentation and a cue associated with no US. Given the striking preference for the safe cue arm, it seems reasonable to conclude that this behavior represents a clear case of instrumental avoidance acquisition. Despite the compelling nature of these data, this "learning conclusion" is offered with considerable hesitancy. Past experience with goldfish has shown that "learning conclusions" have been challenged by unexpected control group performances. Supporting the conclusion in this case, however, is that, since the animal must actively choose on each trial, it serves as its own activity control on a trial-to-trial base; thus, additional activity controls may not be necessary. Since this is one of the first demonstrations of discriminated avoidance using the simultaneous-presentation technique, further work is definitely in order.

\section{REFERENCES}

Scobie, S. R., \& Fallon, D. Operant and Pavlovian control of a defensive shuttle response in goldfish (Cárassius auratus). Journal of Comparative and Physiological Psychology, 1974, 86, 858-866.

STEINER, G. Stimulus control of avoidance learning in fish. Journal of Comparative and Physiological Psychology, 1971, 74, 52-58.

WOODARD, W. T., \& BrtTerman, M. E. Classical conditioning of goldfish in the shuttlebox. Behavior Research Methods \& Instrumentation, 1971, 3, 193-194.

Woodard, W. T., \& Bitterman, M. E. Pavlovian analysis of avoidance conditioning in the goldfish (Carassius auratus). Journal of Comparative and Physiological Psychology, 1973, 82, 123-129.

Zerbolio, D. J., \& Wicrstra, L. L. Goldfish avoidance acquisition: Is the process classical, instrumental, or phototaxis? Bulletin of the Psychonomic Society, 1978, 11, 321-323. (a)

Zerbolio, D. J., \& Wickstra, L. L. Does elimination of a negative phototaxis eliminate CAR acquisition in goldfish? Bulletin of the Psychonomic Society, 1978, 11, 324-326. (b)

Zerbolio, D. J., \& Wickstra, L. L. Passive avoidance in goldfish: Lack of evidence for stimulus specificity. Bulletin of the Psychonomic Society, 1978, 12, 15-17. (c)

Zerbolio, D. J., \& Wickstra, L. L. Discriminated response patterning in goldfish with successive presentation and explicitly unpaired pseudoconditioning procedures. Bulletin of the Psychonomic Society, 1979, 13, 307-310.

(Received February 2, 1979.) 